\title{
Surface Centroid TOA Location Algorithm for VLC System
}

\author{
Yuexia Zhang*, Hang Chen, Shuang Chen, Jiacheng Jin \\ Beijing Information Science \& Technology University \\ Information and communication engineering institute, Beijing 100101, China \\ [e-mail: zhangyuexia@bistu.edu.cn] \\ *Corresponding author: Yuexia Zhang
}

Received February 1, 2018; revised July 17, 2018; accepted August 12, 2018;

published January 31, 2019

\begin{abstract}
The demand for indoor positioning is increasing day by day. However, the widely used positioning methods today cannot satisfy the requirements of the indoor environment in terms of the positioning accuracy and deployment cost. In the existing research domain, the localization algorithm based on three-dimensional space is less accurate, and its robustness is not high. Visible light communication technology (VLC) combines lighting and positioning to reduce the cost of equipment deployment and improve the positioning accuracy. Further, it has become a popular research topic for telecommunication and positioning in the indoor environment. This paper proposes a surface centroid TOA localization algorithm based on the VLC system. The algorithm uses the multiple solutions estimated by the trilateration method to form the intersecting planes of the spheres. Then, it centers the centroid of the surface area as the position of the unknown node. Simulation results show that compared with the traditional TOA positioning algorithm, the average positioning error of the surface centroid TOA algorithm is reduced by $0.3243 \mathrm{~cm}$ and the positioning accuracy is improved by $45 \%$. Therefore, the proposed algorithm has better positioning accuracy than the traditional TOA positioning algorithm, and has certain application value.
\end{abstract}

Keywords: TOA algorithm, centroid of surface, visible light communication, indoor location, three-dimensional space.

This research was supported by the National Natural Science Foundation of China(No.51334003,No.61473039), the Introduction and Training Program of high level talents in Beijing municipal colleges and Universities (No.CIT\&TCD201504058), and the Beijing Information Science and Technology University Graduate Education Quality Engineering Project (No.5121724107). 


\section{Introduction}

The position estimation method can be divided into two types: outdoor and indoor location methods. In the open outdoor environment, the widely used location methods are mainly global navigation satellite system (GNSS), infrared location, ultrasonic location, radar location, radio frequency (RF) localization, and so on. The application of GNSS has been very mature, and it has primarily four commonly used systems, namely, the global positioning system (GPS), a commonly used GLONASS satellite navigation system (GLONASS), Galileo satellite positioning system (GALILEO), and the Beidou satellite navigation system (BDSNS). However, the applications of these systems are severely restricted by high deployment costs and high energy consumption [1]-[4]. Moreover, due to the penetration and partition loss, the GNSS cannot reach the ideal location precision in the city, tunnel, or interior environments [5]. GNSS is recognized to be the legacy system for outdoor environments and, to a great extent, is one of the most accurate sources of position information when it is available. However, its operation in indoor or obstructed environments is infeasible [6].

Indoor localization has gained increasing interest in the last few years because people spend $89 \%$ of their time in indoor environments [7][8]. In order to overcome the shortcoming of GNSS, many scholars have proposed a number of non-GNSS positioning methods based on infrared and ultrasonic signals, radar, and so on. Although these technologies can provide higher positioning accuracy, they need to deploy specific facilities. The main methods of RF include cellular network, wireless LAN (WLAN, Wi-Fi) and wireless sensor network (WSN). These methods have been extensively research on the basis of the existing communication infrastructure [9]-[14]. However, the positioning accuracy of these indoor positioning technologies ranges from tens of centimeters to meters. Such positioning accuracy is not suitable for applications in which high positioning accuracy is needed, such as in hospitals, nursing homes, shopping centers, and military training fields. Therefore, a high precision indoor positioning technology is one of the hotspots in current research. A schematic of the current positioning method is shown in Fig. 1.

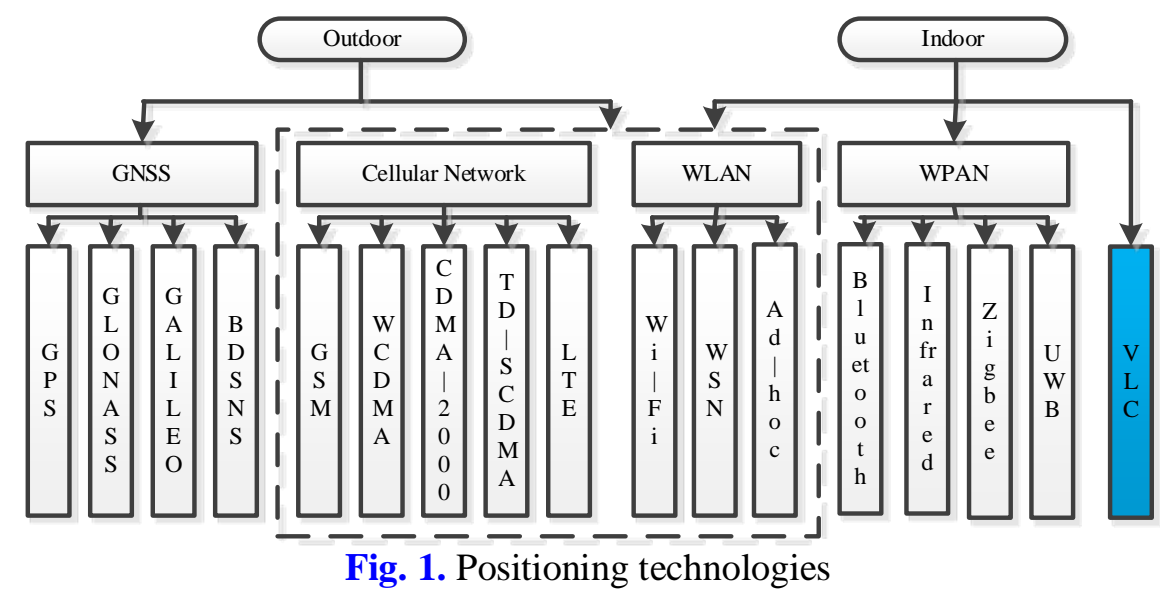

Owing to the rapid development of communication networks, the visible light communication technology (VLC) based on light-emitting diodes (LED) has been 
developing rapidly, and it reflects the characteristics of communication technology, which is different from conventional RF based communication techniques [15]-[21]. First, this communication technology uses flashing light signals rather than infrared and ultrasonic signals. Second, the technology can combine communication with day-to-day lighting. Because of these two characteristics, it demonstrates many advantages compared with RF technology, such as low cost of equipment, easy to deploy equipment, and low signal radiation. Owing to these advantages, VLC technology has become a powerful candidate for next generation wireless networks, and more attention has been paid to location-based services. Unlike in the RF technology, the optical signal is not affected by electromagnetic interference, which makes the positioning system based on VLC achieve higher positioning accuracy. However, because of the propagation characteristics of optical signals, the VLC-based positioning system is not suitable for long-range determination. Moreover, the performance of the system is also easily affected by noise sources such as granular noise, thermal noise, and so on [22]-[24]. The research breakthroughs of visible light communication technology and the popularity of lighting equipment have led to increasing attention on the research of indoor positioning based on VLC. In order to achieve better system performance with low cost, high location accuracy, and other advantages, researchers have made continuous efforts regarding this concept and proposed several technologies [8].

The commonly used ranging and positioning algorithms based on VLC technology include received signal strength (RSS), arrival time (TOA), arrival time difference (TDOA) and arrival angle (AOA). In [25], an indoor location method, which combines the visible light of LED with the indoor location by using multiple optical receivers, is proposed. This method involves an algorithm for changing the transmission distance and angle by using the received signal intensity indicator (RSSI). In addition, in order to estimate the position, multiple optical receivers are used to receive the transmitter position code. In [26], an indoor location system based on the RSS fingerprint of the design criteria is presented; a general probability model is proposed, and the probability that a user can locate in a certain size area is given. Moreover, according to a recently built fingerprint library, the best fingerprint reporting strategy is proposed, which can achieve the best location accuracy and the given reliability and quantity measurement. In [27], an indoor positioning system using white light LED is analyzed; the system uses the modulation signal sent by the LED as the basis for the TOA distance, based on the estimation of the intensity modulation window sine signal and Cramer-Rao bound (CRB) estimation theory to calculate the limit of accuracy. The method in [28] uses TOA to measure the relative position relationship between two locators. Because of the special situation of the TOA ranging error, the indoor-positioning-based distribution model cannot be incorporated using a traditional fixed anchor; a new relative positioning model based on the alpha stable distribution is thus proposed. In [29], a wireless indoor location method based on the TDOA location algorithm in the context of a light emitting diode (LED) is proposed; this method assigns the frequency address to each LED lamp and transmissions through the light radiation of the LED. The positioning accuracy of the indoor space is less than $1 \mathrm{~cm}$ in the range of $5 m \times 5 m \times 3 m$. In [30], Li S,and et al. propose an algorithm considering the correlation between TDOA measurements involving NLOS errors, and integrate the existing TDOA measurement methods. A statistical ranging error model based on experience distance data is proposed, which greatly improves the positioning accuracy. A new concept is proposed in [31] combining the optical wireless visible light communication with three-dimensional indoor positioning by using a single transmitter and multiple tilted light receivers. The channel link is modeled by the characteristic data of the transmitter and receiver. The proposed 3-D positioning algorithm is based on gain difference, 
which is a function of the angle of arrival and the received signal strength. In [32], a three-dimensional indoor location scheme using a single beacon is proposed, which can overcome the limitation of multiple LED beacons involved in one location process. The scheme uses a mixed location algorithm of RSS and AOA to estimate the three-dimensional position. While AOA-based approaches require the direction information of LED transmitters, RSS-based localization considers the captured power from LED transmitters. Although various studies have been performed on VLC systems, more research is required to improve the estimation accuracy.

In this paper, a surface centroid TOA location algorithm (SC-TOA) for VLC systems is proposed. The proposed algorithm uses the multiple solutions estimated by the three-edge localization method to form the intersecting surfaces of spatial spheres. Then, the centroid of the surface region is taken as the unknown node position. The positioning accuracy of the algorithm is better than that of the traditional TOA positioning algorithm. The simulation results show that the average positioning error of the SC-TOA location algorithm is reduced by $0.3243 \mathrm{~cm}$ compared with that in the traditional TOA positioning algorithm. The positioning accuracy is improved by $45 \%$, .

The remaining paper is arranged as follows: Section 2 details the VLC indoor positioning model. Section 3 describes the SC-TOA location algorithm in detail. Section 4 presents the simulation results. Finally, the conclusions are presented.

\section{The system model}

To demonstrate the proposed scheme, we consider an indoor environment of dimensions $5 m \times 5 m \times H$, as shown in Fig. 2. The height of the room, H, is known to us. The indoor VLC environment was composed of N LED arrays located on the ceiling of the room as reference nodes $(\mathrm{RN})$. The white LED light reference nodes are denoted as $S_{1}, S_{2} \cdots S_{i}(i=1 \cdots N)$ in the figure. The coordinates of the sources are known, and are given by $\left(x_{1}, y_{1}, z_{1}\right) 、\left(x_{2}, y_{2}, z_{2}\right) \cdots\left(x_{i}, y_{i}, z_{i}\right)(i=1 \cdots N)$. PN is the node to be located and can be anywhere in the room. Its coordinates are defined as $(x, y, z)$ and are unknown.

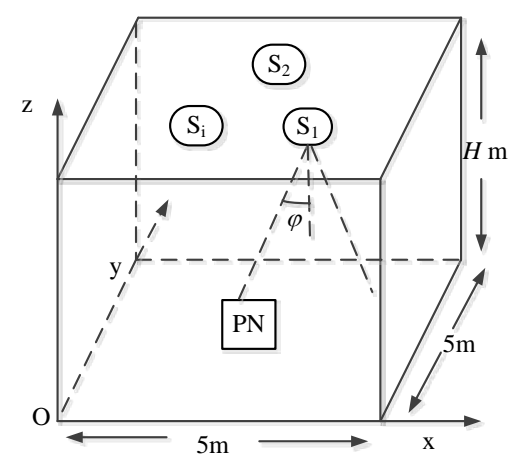

Fig. 2. VLC indoor location model

The RN node can communicate with the PN node through a VLC link. The VLC system communication link is shown in Fig. 3. In Fig. 3 the white LED light source nodes can communicate with the destination node through the VLC link. The modulated optical signal emitted by the source $\mathrm{S}$ is $X(t)$ and the signal received at the destination node using 
photodiodes is $Y(t)$.

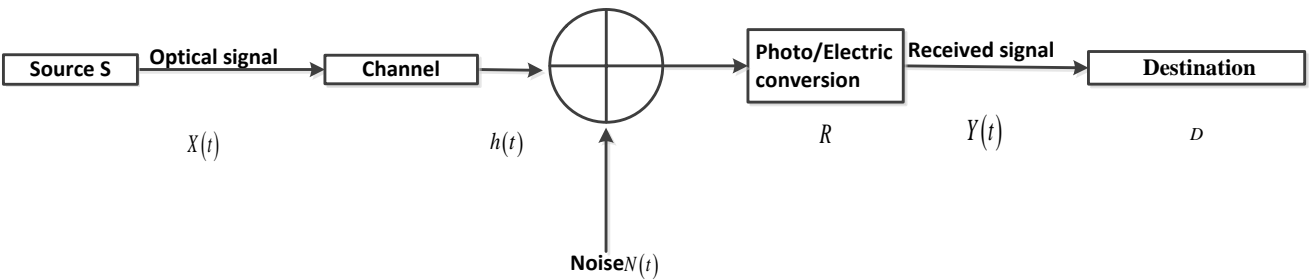

Fig. 3. VLC system communication link

The most common modulation method in optical wireless communication systems is on-off keying modulation (OOK), which uses instantaneous power [33] [34]. The most commonly used wireless optical reception technology is a photo detector that transforms the received light signals into electrical signals. In such situations, the VLC indoor location system will encounter critical interference problems when the LED arrays located on the ceiling of the room overlap. To avoid this problem, each LED uses pulse position modulation (PPM) [35] [36], in which each transmitter emits signals in a given time slot, as shown in Fig. 4. Each signal consist of data as well as the source's location information. The signals received by the destination from the various sources will not interference each other and can be used to analyze signal characteristics.

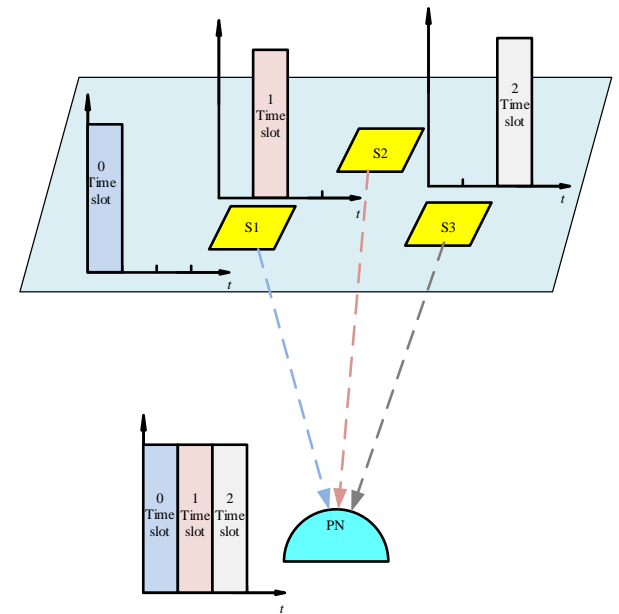

Fig. 4. VLC PPM modulation

We consider a source and destination, as represented above, in an indoor environment under a guaranteed line of sight (LOS) condition. If the distance between the source and destination is much larger than the area $A_{r}$, then the received irradiance is approximately constant over the surface of the receiver. Furthermore, all signals are considered to arrive at the same time at the receiver. Thus, $Y(t)$ can be expressed as

$$
Y(t)=R X(t) \cdot h(t)+N(t)
$$

where, $R$ is the photoelectric sensor conversion efficiency and $h(t)$ is the channel impulse response. The channel impulse of the VLC system can be represented as: 


$$
\begin{aligned}
& h(t)=\frac{A_{r}(m+1)}{2 \pi d^{2}} \cos ^{m}(\varphi) T_{s}(\psi) g(\psi) \\
& \cos (\psi) \delta\left(t-\frac{d}{c}\right), 0 \leq \psi \leq \text { FOV }
\end{aligned}
$$

where $A_{r}$ is the receiving area of the photo detector, $m=-\ln 2 / \ln \left(\cos \varphi_{1 / 2}\right), d$ is the distance between the source and destination, $\varphi$ is the radiation angle, $T_{s}(\psi)$ is the gain of the optical filter sink, $g(\psi)$ is the gain of an optical concentrator, $c=3 \times 10^{8} \mathrm{~m} / \mathrm{s}$ is the speed of light, $\psi$ is the receiving angle, FOV is the field angle of the source, $\mathrm{M}$ represents Lambert's radiation coefficient, and $\varphi_{1 / 2}$ refers to the half power angle of the LED light source. Noise is one of the main factors that affect the accuracy of the location algorithm. It is mainly composed of thermal noise, shot noise from background radiation and so on [37]-[39]. According to [39], the noise can be modeled as a zero-mean Gaussian process. So $N(t)$ is Gaussian white noise.

\section{Proposed SC-TOA algorithm}

\subsection{Traditional TOA positioning algorithm}

The traditional TOA positioning algorithm obtains the transmission time of optical signals by measuring from a known reference node to a point to be measured, and calculates the distance between the measured point and the known source. The algorithm uses the three known sources as the centers of spheres; the point of intersection of the three spheres gives the coordinates of the point to be measured. A diagram of the principle of the traditional TOA positioning algorithm is shown in Fig. 5.

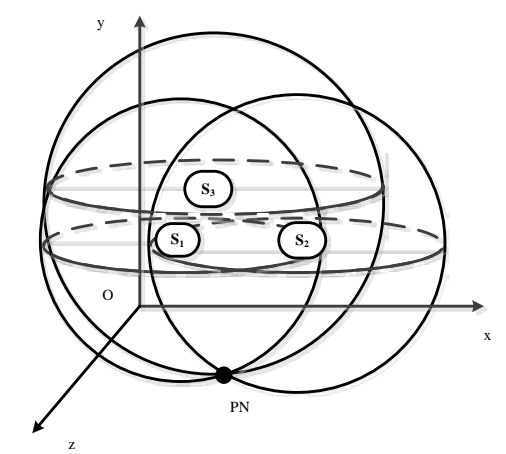

Fig. 5. Principle of the traditional TOA positioning algorithm

The distance between the LED light reference node and the PN node to be located can be calculated by the lower form:

$$
d_{i}=c \cdot t_{i} \quad i=1,2,3
$$

In equation (3), $d_{i}(i=1,2,3)$ represents the distance between the LED light reference node and the PN node, $t_{i}=T_{i}-T_{0}(i=1,2,3)$ represents the transmission time of the light signal from the light source to the PN node to be located, $T_{0}$ represents the emission time of the light signal at the LED transmitter, and $T_{i}$ represents the receiving time of the optical signal 
received by the $\mathrm{PN}$ receiver.

The distance between the point to be located and the three light sources can then be calculated to be $d_{1} 、 d_{2} 、 d_{3}$. It satisfies the following equation:

$$
\left\{\begin{array}{l}
d_{1}=c \cdot t_{1} \\
d_{2}=c \cdot t_{2} \\
d_{3}=c \cdot t_{3}
\end{array}\right.
$$

According to the known coordinates, the spherical positioning formula is constructed as follows:

$$
\left\{\begin{array}{l}
\left(x_{1}-x\right)^{2}+\left(y_{1}-y\right)^{2}+\left(z_{1}-z\right)^{2}=d_{1}^{2} \\
\left(x_{2}-x\right)^{2}+\left(y_{2}-y\right)^{2}+\left(z_{2}-z\right)^{2}=d_{2}{ }^{2} \\
\left(x_{3}-x\right)^{2}+\left(y_{3}-y\right)^{2}+\left(z_{3}-z\right)^{2}=d_{3}{ }^{2}
\end{array}\right.
$$

The PN coordinates can then be calculated.

\subsection{The principle of the SC-TOA algorithm}

In a VLC indoor location environment, as shown in Fig. 2, the LED reference nodes are placed on the roof to satisfy the equation $z_{1}=z_{2}=z_{3}=H$. Therefore, according to equation (5), the coordinates of the PN nodes can be expressed as follows

$$
\left\{\begin{array}{l}
d_{1}^{2}=\left(x_{1}-x\right)^{2}+\left(y_{1}-y\right)^{2}+(H-z)^{2} \\
d_{2}^{2}=\left(x_{2}-x\right)^{2}+\left(y_{2}-y\right)^{2}+(H-z)^{2} \\
d_{3}^{2}=\left(x_{3}-x\right)^{2}+\left(y_{3}-y\right)^{2}+(H-z)^{2}
\end{array}\right.
$$

Equation (6) can be changed into the following matrix form:

$$
P X=Q
$$

where

$$
\begin{gathered}
P=\left[\begin{array}{cc}
x_{2}-x_{1} & y_{2}-y_{1} \\
x_{3}-x_{1} & y_{3}-y_{1} \\
x_{3}-x_{2} & y_{3}-y_{2}
\end{array}\right] \\
X=\left[\begin{array}{l}
x \\
y
\end{array}\right] \\
Q=\left[\begin{array}{c}
\left(d_{1}^{2}-d_{2}^{2}+x_{2}^{2}+y_{2}^{2}-x_{1}^{2}-y_{1}^{2}\right) / 2 \\
\left(d_{1}^{2}-d_{3}^{2}+x_{3}^{2}+y_{3}^{2}-x_{1}^{2}-y_{1}^{2}\right) / 2 \\
\left(d_{2}^{2}-d_{3}^{2}+x_{3}^{2}+y_{3}^{2}-x_{2}^{2}-y_{2}^{2}\right) / 2
\end{array}\right] .
\end{gathered}
$$


The least squares method is used to solve matrix equation (7). First, the residual value is defined as $f=Q-P X$. The residual value satisfies equation (8).

$$
f^{2}=(Q-P X)^{2}=(Q-P X)^{T}(Q-P X)
$$

Therefore, the problem is transformed into solving the minimum value of the symbol as $f^{2}$. The algorithm requires derivation of equation (8) and makes the derivative zero. The solution of $X=[\mathrm{x}, y]^{T}$ can be obtained as follows:

$$
X=\left(P^{T} P\right)^{-1} P^{T} Q
$$

When the result is substituted into equation (6), the PN coordinates can be obtained.

Ideally, as long as the source is not collinear, equation (9) must have a unique solution, and the three spheres in Fig. 5 will intersect at one point. However, because of measurement error and the influence of LED SAHP, the three spheres intersect at more than one point, and form a curved surface area. This shows that equation (9) has multiple solutions, and the surface area is bounded by these solutions.

Therefore, in order to estimate the location of the point to be located, PN, the centroid of the surface area is used to replace the position of PN. The centroid of the surface can be obtained as follows:

$$
\left\{\begin{array}{c}
\bar{x}=\frac{\iint_{\Sigma} x \rho(x, y, z) d S}{\iint_{\Sigma} \rho(x, y, z) d S} \\
\bar{y}=\frac{\iint_{\Sigma} y \rho(x, y, z) d S}{\iint_{\Sigma} \rho(x, y, z) d S} \\
\bar{z}=\frac{\iint_{\Sigma} z \rho(x, y, z) d S}{\iint_{\Sigma} \rho(x, y, z) d S}
\end{array}\right.
$$

where $S=\iint_{\Sigma} d S$ represents the area of the surface and $\rho$ represents density. The density is the density of air at room temperature of $20{ }^{\circ} \mathrm{C}$ at standard atmospheric pressure, and its value is $1.205 \mathrm{~kg} / \mathrm{m}^{3}$.

The algorithm uses (10) to calculate the coordinates of the point to be located PN and its coordinate is $(\bar{x}, \bar{y}, \bar{z})$. 


\section{Simulation results and analysis}

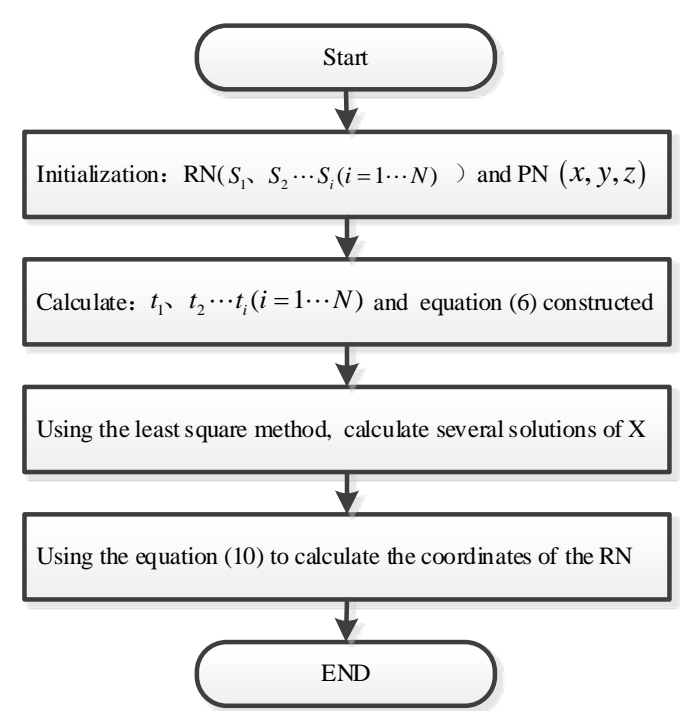

Fig. 6. Simulation flowchart

We conducted simulations to evaluate the performance of the proposed system. The simulation environment setup is shown in Fig. 2. The monitored region was set to an indoor environment measuring $5 m \times 5 m \times 3 \mathrm{~m}$. Specifically, there were several reference nodes and a PN node to be located. The simulation flowchart is shown in Fig. 6. First, we placed RN nodes on the ceiling of the room such that they were not in the same line. The number of the RN nodes was N. PN node could be located anywhere in the room. Then, the communication time between each PN node and the RN node was calculated, and equation (6) was constructed. Using equations (6)-(9) and the least squares method, we could calculate several solutions for $\mathrm{X}$. Finally, we used equation (10) to calculate the coordinates of the RN.

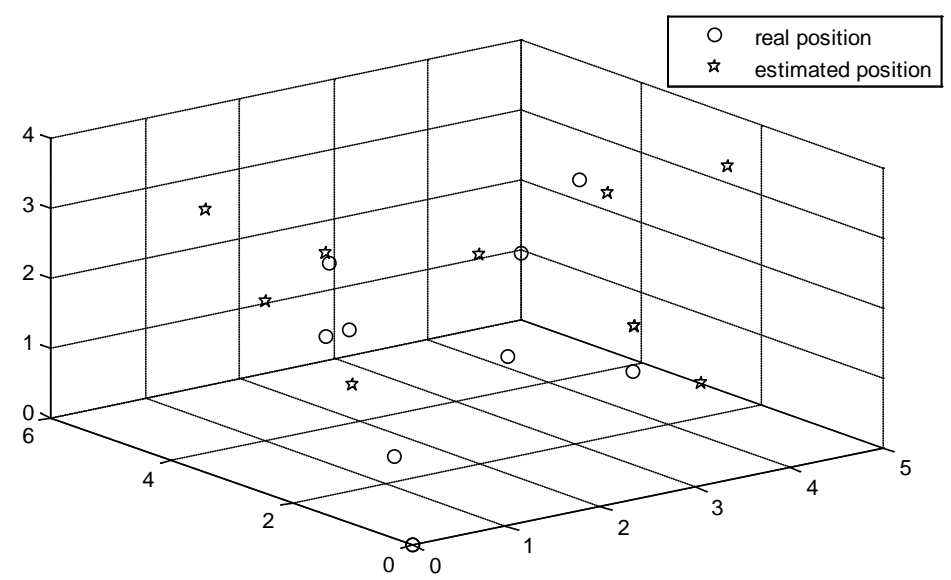

Fig. 7. Real and estimated PN positions

The real position of the PN node and the position estimated by the SC-TOA algorithm are shown in Fig. 7. In Fig. 7, the $x, y$, and $z$ axes represent the length, width, and height of the model room, respectively. The circle represents the real position of the PN node. 
Five-pointed star expresses the estimated position of the PN node. As can be seen, there is a positioning error between the estimated and real positions.

The localization performance of the SC-TOA algorithm is shown in Fig. 8. In Fig. 8, the $x$ and y axes represent the length and width of the room model, respectively. The distribution of the positioning error over the room for the indoor VLC system can be seen from the figure. In general, the positioning error in the room is relatively small. However, in the corners of the room, the positioning error is relatively large, because the $\mathrm{RN}$ node receives multipath signals.

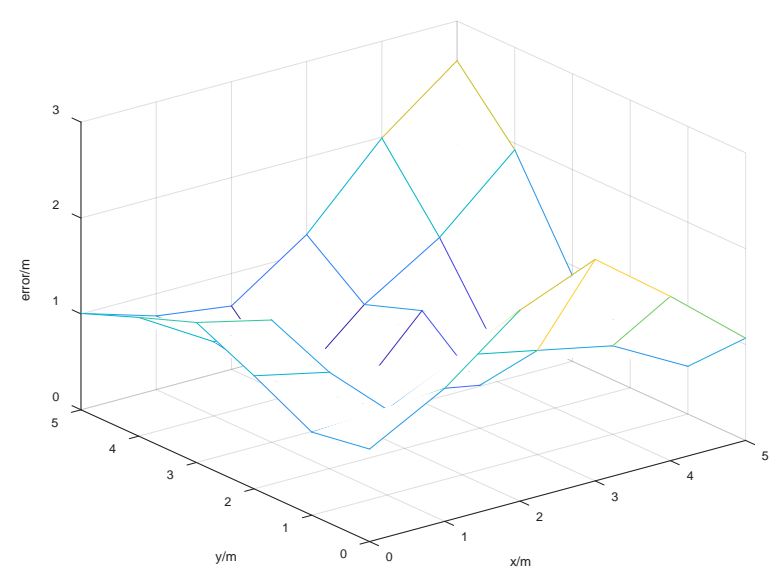

Fig. 8. The location error of the SC-TOA algorithm

The positioning errors of different algorithms are shown in Fig. 9. The abscissa represents the number of positions and the ordinate represents the positioning error. In Fig. 9, the curve labeled with hollow circles represents the traditional TOA algorithm. The curve labeled with five pointed stars represents the SC-TOA algorithm. The error of the traditional TOA algorithm is lower than $0.9 \mathrm{~cm}$, whereas the error of the SC-TOA algorithm is lower than 0.6 $\mathrm{cm}$, indicating that the latter has higher positioning accuracy.

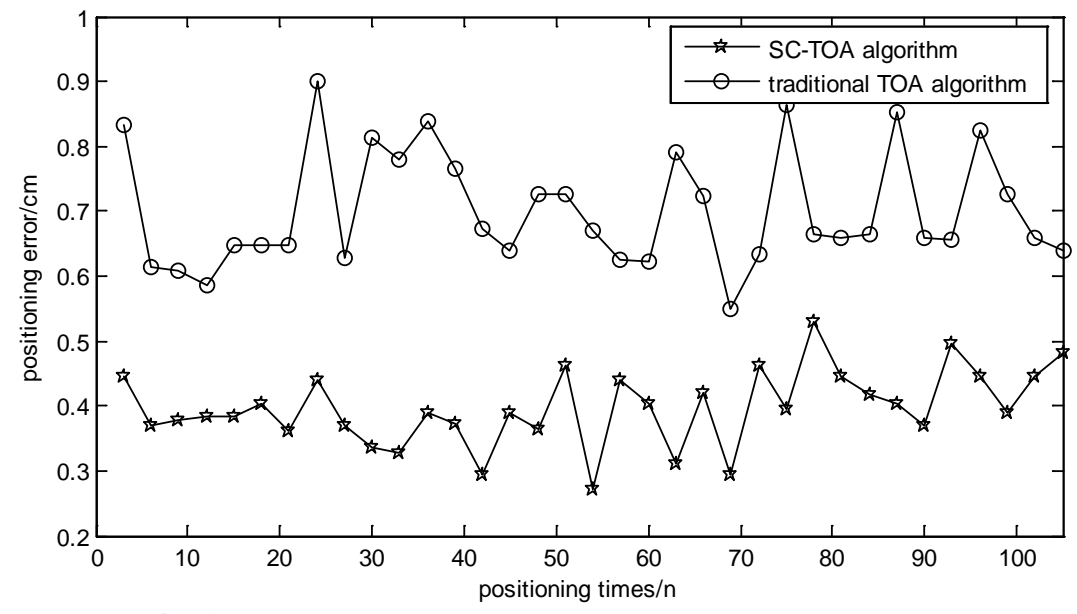

Fig. 9. Comparison of location error for different algorithms

Fig. 10, shows the relationship between the average location error and the number of reference nodes for the two different location algorithms. The abscissa represents the number of sources, and the ordinate represents the mean location error. In Fig. 10, the 
curve noted by the five-pointed stars represents the SC-TOA algorithm, and the curve noted by the hollow circles represents the traditional TOA positioning algorithm. The average location error of the location algorithms decreases as the number of sources increases. Moreover, the average positioning error of the SC-TOA algorithm is lower than that of the traditional TOA algorithm in the case of the same number of reference nodes. It can be seen that the average positioning error of the SC-TOA algorithm is lower than that of the traditional TOA algorithm by $0.3243 \mathrm{~cm}$, and its positioning accuracy is improved by $45 \%$.

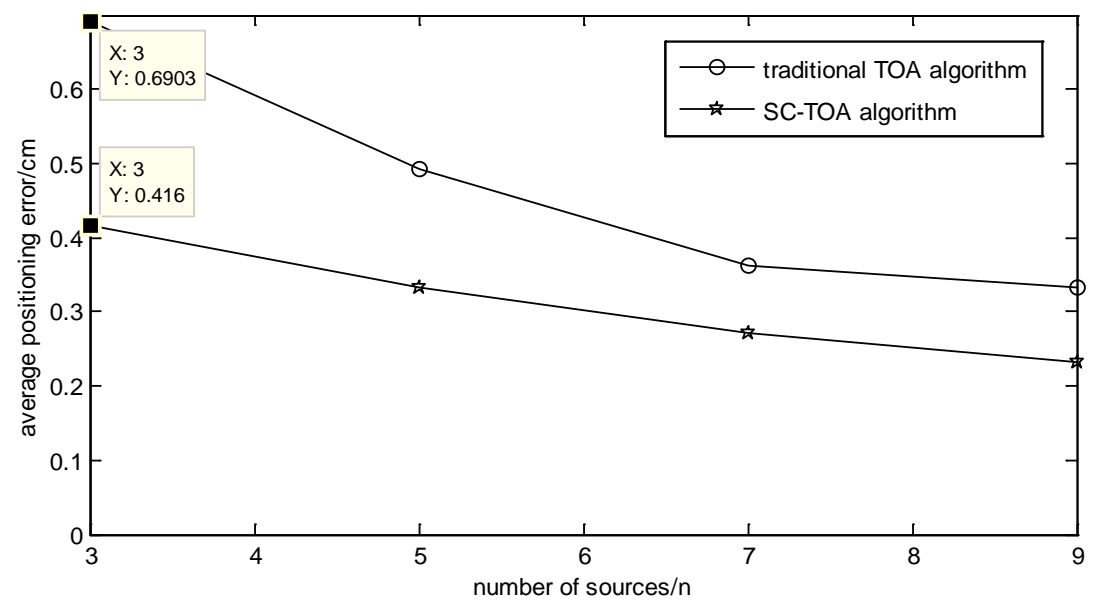

Fig. 10. Comparison of location error and source number

\section{Conclusion}

VLC technology based on LEDs is considered to be the most promising communication technology for the future, because it can be used for both lighting and communication. Compared to traditional wireless communication at radio frequencies, VLC has several advantages such as cost-effectiveness, immunity to electromagnetic interference, and a larger modulation bandwidth. Therefore, a VLC system could be deployed in indoor environments for both communication and positioning. In this work, a SC-TOA algorithm based on a VLC system is proposed. The algorithm uses the multiple solutions estimated by the trilateration method to form the intersecting planes of the spheres. It then calculates the centroid of the surface area as the position of the unknown node. The simulation results show that the average positioning error of the SC-TOA positioning algorithm is lower than that of the traditional TOA positioning algorithm by $0.3243 \mathrm{~cm}$, and the positioning accuracy is improved by $45 \%$. The complexity of the algorithm is relatively low, and it exhibits improved positioning accuracy. It has good potential for application in hospitals, shopping centers, apartments for the elderly, and other indoor environments.

\section{Reference}

[1] Büyükçorak, Saliha, and G. K. Kurt, "A Bayesian Perspective on RSS Based Localization for Visible Light Communication with Heterogeneous Networks Extension," IEEE Access, vol. 5, pp. 17487-17500, August 29, 2017. Article (CrossRef Link). 
[2] Park, Jong Kang, et al, "Hadamard Matrix Design for a Low-Cost Indoor Positioning System in Visible Light Communication,” IEEE Photonics Journal, vol. 9, no. 2, pp. 1-10, February 09, 2017. Article (CrossRef Link).

[3] Zheng D, Chen G, Farrell J A, "Joint Measurement and Trajectory Recovery in Visible Light Communication,” IEEE Transactions on Control Systems Technology, vol. 25, no. 1, pp.247-261, May 12, 2016. Article (CrossRef Link).

[4] Li X, Wu L, Liu Z, et al, "Design and Characterization of Active Matrix LED Microdisplays With Embedded Visible Light Communication Transmitter,” Journal of Lightwave Technology, vol. 34, no. 14, pp.3449-3457, May 04, 2016. Article (CrossRef Link).

[5] Gu W, Aminikashani M, Deng P, et al, "Impact of Multipath Reflections on the Performance of Indoor Visible Light Positioning Systems,” Journal of Lightwave Technology, vol. 34, no. 10, pp.2578-2587, March 11, 2016. Article (CrossRef Link).

[6] Dardari D, Closas P, Djurić P M, "Indoor Tracking: Theory, Methods, and Technologies," IEEE Transactions on Vehicular Technology, vol. 64, no. 4, pp.1263-1278, February 13, 2015. Article (CrossRef Link).

[7] Elhamshary M, Basalmah A, Youssef M, “A Fine-Grained Indoor Location-Based Social Network,” IEEE Transactions on Mobile Computing, vol. 16, no. 5, pp.1203-1217, July 14, 2017. Article (CrossRef Link).

[8] Luo J, Fan L, Li H, "Indoor Positioning Systems Based on Visible Light Communication: State of the Art,” IEEE Communications Surveys \& Tutorials, vol. 19, no. 4, pp.2871-2893, August 23, 2017. Article (CrossRef Link).

[9] Abu-Shaban Z, Zhou X, Abhayapala T D, “A Novel TOA-Based Mobile Localization Technique Under Mixed LOS/NLOS Conditions for Cellular Networks,” IEEE Transactions on Vehicular Technology, vol. 65, no.11, pp.8841-8853, January 12, 2016. Article (CrossRef Link).

[10] He S, Chan S H G, "Wi-Fi Fingerprint-Based Indoor Positioning: Recent Advances and Comparisons,” IEEE Communications Surveys \& Tutorials, vol. 18, no. 1, pp.466-490, August 03, 2015. Article (CrossRef Link).

[11] Li X, Cai X, Hei Y, et al, "NLOS identification and mitigation based on channel state information for indoor WiFi localisation,” Iet Communications, vol. 11, no. 4, pp.531-537, March 09, 2017. Article (CrossRef Link).

[12] Ruiz A R J, Granja F S, “Comparing Ubisense, BeSpoon, and DecaWave UWB Location Systems: Indoor Performance Analysis,” IEEE Transactions on Instrumentation \& Measurement, vol. 66, no. 8, pp.2106-2117, April 03, 2017. Article (CrossRef Link).

[13] Yang P, "PRLS-INVES: A General Experimental Investigation Strategy for High Accuracy and Precision in Passive RFID Location Systems,” IEEE Internet of Things Journal, vol. 2, no. 2, pp.159-167, December 04, 2014. Article (CrossRef Link).

[14] Liu S, Jiang Y, Striegel A, "Face-to-Face Proximity EstimationUsing Bluetooth On Smartphones," IEEE Transactions on Mobile Computing, vol. 13, no. 4, pp.811-823, March 27, 2013. Article (CrossRef Link).

[15] Hussain B, Che F, Zhang F, et al, “A fully integrated IEEE 802.15.7 visible light communication transmitter with on-chip 8-W 85\% efficiency boost LED driver,” in Proc. of the 2015 Symposium on VLSI Circuits, pp.216-217, June 17-19, 2015. Article (CrossRef Link).

[16] Hussein A T, Alresheedi M T, Elmirghani J M H, "20 Gb/s Mobile Indoor Visible Light Communication System Employing Beam Steering and Computer Generated Holograms,” Journal of Lightwave Technology, vol. 33, no. 24, pp.5242-5260, October 26, 2015 Article (CrossRef Link).

[17] Şahin A, Eroğlu Y S, İsmail Güvenç, et al, "Hybrid 3-D Localization for Visible Light Communication Systems," Journal of Lightwave Technology, vol. 33, no.22, pp.4589-4599, September 09, 2015. Article (CrossRef Link).

[18] Chen Y A, Chang Y T, Tseng Y C, et al, “A Framework for Simultaneous Message Broadcasting Using CDMA-Based Visible Light Communications,” IEEE Sensors Journal, vol. 15, no. 12, pp.6819-6827, August 03, 2015. Article (CrossRef Link). 
[19] Yasir M, Ho S W, Vellambi B N, "Indoor Positioning System Using Visible Light and Accelerometer,” Journal of Lightwave Technology, vol. 32, no. 19, pp.3306-3316, July 31, 2014. Article (CrossRef Link).

[20] Mousa F I K, Almaadeed N A S A, Busawon K K, et al, "Secure MIMO Visible Light Communication System Based on User's Location and Encryption," Journal of Lightwave Technology, vol. 35, no. 24, pp.5324-5334, October 09, 2017. Article (CrossRef Link).

[21] Wang C, Wang L, Chi X, et al, “The Research of Indoor Positioning Based on Visible Light Communication,” China Communication, vol. 12, no. 8, pp.85-92, 2015. Article (CrossRef Link).

[22] Bingcheng Zhu, Julian Cheng, Yongjin Wang, et al, "Three-Dimensional VLC Positioning Based on Angle Difference of Arrival With Arbitrary Tilting Angle of Receiver,” IEEE Journal on Selected Areas in Communications, vol. 36, no. 1, pp.8-22, December 01, 2017. Article (CrossRef Link).

[23] Saab S S, Saab K K, “A Positioning System for Photodiode Device Using Collocated LEDs,” IEEE Photonics Journal, vol. 8, no. 5, pp.1-14, September 20, 2016.Article (CrossRef Link).

[24] Guzman B G, Serrano A L, Jimenez V P G, "Cooperative optical wireless transmission for improving performance in indoor scenarios for visible light communications," IEEE Transactions on Consumer Electronics, vol. 61, no.4, pp.393-401, November, 2015. Article (CrossRef Link).

[25] Yang S H, Jung E M, Han S K, "Indoor Location Estimation Based on LED Visible Light Communication Using Multiple Optical Receivers,” IEEE Communications Letters, vol. 17, no. 9, pp.1834-1837, July 16, 2013. Article (CrossRef Link).

[26] Tian X, Shen R, Liu D, et al, "Performance Analysis of RSS Fingerprinting based Indoor Localization," IEEE Transactions on Mobile Computing, vol. 16, no. 10, pp.2847-2861, December 26, 2016. Article (CrossRef Link).

[27] Wang T Q, Sekercioglu Y A, Neild A, et al, "Position Accuracy of Time-of-Arrival Based Ranging Using Visible Light With Application in Indoor Localization Systems," Journal of Lightwave Technology, vol. 31, no. 20, pp.3302-3308, September 16, 2013. Article (CrossRef Link).

[28] Wang P, He J, Xu L, et al, "Characteristic Modeling of TOA Ranging Error in Rotating Anchor-Based Relative Positioning,” IEEE Sensors Journal, vol. 17, no. 23, pp.7945-7953, September 28, 2017. Article (CrossRef Link).

[29] Jung S Y, Hann S, Park C S, "TDOA-based optical wireless indoor localization using LED ceiling lamps," IEEE Transactions on Consumer Electronics, vol. 57, no. 4, pp.1592-1597, November, 2011. Article (CrossRef Link).

[30] Li S, Hedley M, Collings I B, et al, “TDOA-Based Localization for Semi-Static Targets in NLOS Environments,” IEEE Wireless Communications Letters, vol. 4, no.5, pp.513-516, June 24, 2015. Article (CrossRef Link).

[31] Yang S H, Kim H S, Son Y H, et al, "Three-Dimensional Visible Light Indoor Localization Using AOA and RSS With Multiple Optical Receivers,” Journal of Lightwave Technology, vol. 32, no. 14, pp.2480-2485, May 30, 2014. Article (CrossRef Link).

[32] Hou Y, Xiao S, Bi M, et al, "Single LED Beacon-Based 3-D Indoor Positioning Using Off-the-Shelf Devices,” IEEE Photonics Journal, vol. 8, no. 6, pp.1-11, December 07, 2016. Article (CrossRef Link).

[33] Bassem Fahs; Jeffrey Chellis,et al, “A 6-m OOK VLC Link Using CMOS-Compatible p-n Photodiode and Red LED,” IEEE Photonics Technology Letters, vol.28,no. 24, pp: 2846 - 2849, 2016. Article (CrossRef Link).

[34] Zunaira Babar; Mohd Azri Mohd Izhar, et al, "Unary-Coded Dimming Control Improves ON-OFF Keying Visible Light Communication,” IEEE Transactions on Communications, vol.66, no.1, pp: 255 - 264, 2018. Article (CrossRef Link).

[35] Mehmet Sönmez, "Simplified and accelerated PPM receivers for VLC systems," IET Optoelectronics, vol. 12, no. 1, pp: 36-43, 2018. Article (CrossRef Link). 
[36] Jong-Ho Yoo; Byung Wook Kim; Sung-Yoon Jung, "Modelling and analysis of M-ary variable pulse position modulation for visible light communications,” IET Optoelectronics,vol. 9, no. 5, pp:184-190, 2018. Article (CrossRef Link).

[37] H. Manor and S. Arnon, "Performance of an optical wireless communication system as a function of wavelength,” Appl. Opt., vol. 42, no. 21, pp. 4285-4294, Jul. 2003. Article (CrossRef Link).

[38] X. Zhang, J. Duan, Y. Fu, and A. Shi, "Theoretical accuracy analysis of indoor visible light communication positioning system based on received signal strength indicator," J. Lightw. Technol., vol. 32, no. 21, pp. 4180-4186, Nov. 2014. Article (CrossRef Link).

[39] T. Komine and M. Nakagawa, "Fundamental analysis for visible-light communication system using LED lights,” IEEE Trans. Consum. Electron., vol. 50, no. 1, pp. 100-107, Feb. 2004. Article (CrossRef Link).

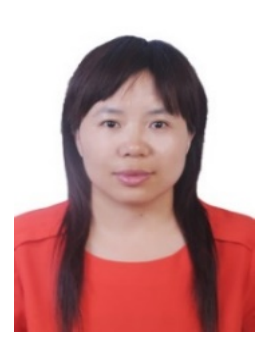

Yuexia Zhang was born in Anyang Village, Henan Province, China in 1978. She received the B.S. degree in detection technology and instrument from Zhengzhou University, Henan, in 2001. She received the M.S. degree and Ph.D. degree in information and communication engineering for Beijing University of Posts and Telecommunications Beijing, in 2008. From 2008 to now, she was an Assistant Professor with the School of Information and Communication Engineering, Beijing Information Science and Technology University. She is the author of three books, more than 20 articles, and more than 10 inventions. Her research interests include wireless cooperative communication technology, ultra-wideband technology, and wireless positioning technology. She holds two patents.

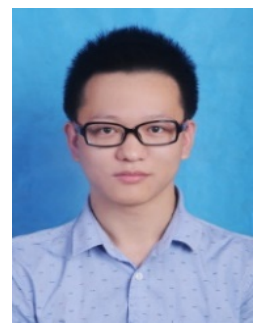

Hang Chen received the B.E in network engineering from Liaoning University of Technology in 2015. He has published papers about routing algorithms and LED half power angle visible light indoor positioning methods and other papers. He is mainly engaged in mobile communication and $5 \mathrm{G}$ positioning.

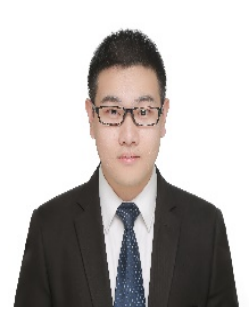

Jiacheng Jin received the B.E.E degrees in electrical engineering from Beijing Information Science and Technology University in 2017. At present, he is a graduate student of Beijing Information Science and Technology University. He is mainly engaged in visible light localization and 5G orientation research. 